\title{
1s2p resonant inelastic X-ray scattering combined dipole and quadrupole analysis method
}

Bagger, Alexander; Haarman, Ties; Molina, Anna Puig; Moses, Poul George; Ishii, Hirofumi; Hiraoka, Nozomu; Wu, Yu-Han; Tsuei, Ku-Ding; Chorkendorff, Ib; De Groot, Frank

Published in:

Journal of Synchrotron Radiation

Link to article, DOI:

$10.1107 / \mathrm{S} 1600577516016933$

Publication date:

2017

Document Version

Publisher's PDF, also known as Version of record

Link back to DTU Orbit

Citation (APA):

Bagger, A., Haarman, T., Molina, A. P., Moses, P. G., Ishii, H., Hiraoka, N., Wu, Y-H., Tsuei, K-D., Chorkendorff, I., \& De Groot, F. (2017). $1 s 2 p$ resonant inelastic X-ray scattering combined dipole and quadrupole analysis method. Journal of Synchrotron Radiation, 24(1), 296-301. https://doi.org/10.1107/S1600577516016933

\section{General rights}

Copyright and moral rights for the publications made accessible in the public portal are retained by the authors and/or other copyright owners and it is a condition of accessing publications that users recognise and abide by the legal requirements associated with these rights.

- Users may download and print one copy of any publication from the public portal for the purpose of private study or research.

- You may not further distribute the material or use it for any profit-making activity or commercial gain

- You may freely distribute the URL identifying the publication in the public portal 
JOURNAL OF

SYNCHROTRON

RADIATION

ISSN 1600-5775

Received 22 June 2016

Accepted 22 October 2016

Edited by R. W. Strange, University of Essex, UK

Keywords: resonant inelestic X-ray scattering; RIXS; X-ray absorption; X-ray emission.

\section{$1 s 2 p$ resonant inelastic X-ray scattering combined dipole and quadrupole analysis method}

\author{
Alexander Bagger, ${ }^{\mathrm{a}, \mathrm{b} *}$ Ties Haarman, ${ }^{\mathrm{b}, \mathrm{c}}$ Anna Puig Molina, ${ }^{\mathrm{b}}$ Poul George Moses, \\ Hirofumi Ishii, ${ }^{d}$ Nozomu Hiraoka, ${ }^{\mathrm{d}}$ Yu-Han Wu, ${ }^{\mathrm{d}}$ Ku-Ding Tsuei, ${ }^{\mathrm{d}}$ Ib Chorkendorff ${ }^{\mathrm{a}}$ \\ and Frank De Groot ${ }^{\mathrm{c}}$
}

\begin{abstract}
${ }^{a}$ Department of Physics, Technical University of Denmark, DK-2800 Lyngby, Denmark, ${ }^{\mathbf{b}}$ Haldor Topsoe A/S, DK-2800 Lyngby, Denmark, ${ }^{\mathbf{C}}$ Department of Inorganic Chemistry and Catalysis, Debye Institute, University of Utrecht, Sorbonnelaan 16, 3584 CA Utrecht, The Netherlands, and dNational Synchrotron Radiation Research Center, SPring-8, Japan. *Correspondence e-mail: bagger.alexander@gmail.com
\end{abstract}

In this study an analysis strategy towards using the resonant inelastic X-ray scattering (RIXS) technique more effectively compared with X-ray absorption spectroscopy (XAS) is presented. In particular, the question of when RIXS brings extra information compared with XAS is addressed. To answer this question the RIXS plane is analysed using two models: (i) an exciton model and (ii) a continuum model. The continuum model describes the dipole pre-edge excitations while the exciton model describes the quadrupole excitations. Applying our approach to the experimental $1 s 2 p$ RIXS planes of $\mathrm{VO}_{2}$ and $\mathrm{TiO}_{2}$, it is shown that only in the case of quadrupole excitations being present is additional information gained by RIXS compared with XAS. Combining this knowledge with methods to calculate the dipole contribution in XAS measurements gives scientists the opportunity to plan more effective experiments.

\section{Introduction}

Resonant inelastic X-ray scattering (RIXS) spectroscopy is an advanced synchrotron technique that combines X-ray absorption spectroscopy (XAS) and X-ray emission spectroscopy (XES). Thus, the RIXS technique is element-selective and measures both the unoccupied and occupied states together with their interference. Here one can probe magnetic and phonon modes (Ament et al., 2011). Experimentally, the RIXS plane is collected by scanning an emission energy window at each absorption energy step through the $K$-, $L$ - or $M$-edge regions, resulting in a two-dimensional RIXS plane. RIXS has proven successful in giving insight into bulk materials, nanoparticles and molecular metal complexes (Smolentsev et al., 2011; Glatzel et al., 2010; Garino et al., 2012; De Groot \& Kotani, 2008). However, there can be systems where RIXS does not bring additional information compared with XAS.

In this paper we address the question of when it is beneficial to measure $1 s 2 p$ RIXS, where the $1 s 2 p$ labels the $K$-edge excitation and the $K_{\alpha}$ decay. As for all experimental techniques, the important point is not the measurement itself but how we obtain information from the experiment.

In the theory section, we present two known models for RIXS data analysis: (i) the exciton model, describing the excitation to an exciton state, and (ii) the continuum model, describing the excitation to a band state.

First we show for an arbitrary system how RIXS spectral features are generated with the continuum model. This will show the general XAS and XES convoluting origin of the 
RIXS measurement. Next, the two models are applied to real RIXS data: the $\mathrm{VO}_{2}$ (newly recorded) and $\mathrm{TiO}_{2}$ systems. Finally, a methodical experimental RIXS approach is suggested based on the results.

\section{Theory}

The mathematical formulation of the RIXS process is described by the Kramers-Heisenberg equation, which is a second-order process. The Kramers-Heisenberg equation can be compared with the Golden Rule describing the XAS process. The Kramers-Heisenberg equation is formulated as (De Groot \& Kotani, 2008)

$$
\begin{aligned}
\frac{\mathrm{d}^{2} \sigma}{\mathrm{d} \Omega \mathrm{d} \omega}= & r_{0}^{2} \frac{\omega}{\Omega} \sum_{j}\left|\sum_{i} \frac{\left\langle j\left|T_{2}\right| i\right\rangle\left\langle j\left|T_{1}\right| g\right\rangle}{E_{g}+\hbar \Omega-E_{\mathrm{i}}+i \Gamma_{\mathrm{i}}}\right|^{2} \\
& \times \delta\left(E_{g}+\hbar \Omega-E_{j}-\hbar \omega\right),
\end{aligned}
$$

where $\sigma$ is the scattering cross-section, $r_{0}^{2}$ is the electron radius, $\hbar \omega$ is the emission photon energy, $\hbar \Omega$ is the absorption photon energy, $T_{1}$ and $T_{2}$ are the transition operators and $\Gamma_{\mathrm{i}}$ decribes the lifetime broadening of the intermediate state. The labelling $g, i$ and $j$ denotes the ground state, intermediate state and final state, respectively. $E$ describes the energy of the $|g\rangle$, $\langle i|| i$,$\rangle and \langle j|$ states including both photon and full electronic description. To calculate RIXS planes the equation is simplified, as follows:

(i) In the exciton model: the charge transfer multiplet (CTM) model applies the equation to a limited electronic system (Stavitski \& de Groot, 2010). The approximations in this model can be listed as: (1) calculation of electron-electron interaction of an isolated atom in the Hartree-Fock approximation, which is scaled to atomic data, and includes the spinorbit term; (2) local environment ligand charge fluctuations are included by a perturbing crystal field term and charge transfer excitations; and (3) transition energies are not calculated but tabulated values are used instead. This essentially means that for a $K$-edge calculation, within this framework, the calculation results in a local quadrupole transition calculation. In general, it must be noted that calculating the transition energies from first principles is difficult due to incorrect description of the core hole screening (Juhin et al., 2010). The multiplet model has, among others, been successfully applied to describe hard X-ray RIXS features of Fe complexes (de Groot et al., 2005) and CoO (Kurian et al., 2013).

(ii) In the continuum model: instead of applying the Kramers-Heisenberg equation to electronic calculations, it was simplified with two approximations by Tulkki \& Åberg (1982). The first approximation assumes one electron states and the second assumes that the intermediate and final states are orthogonal to each other, which results in the following equation:

$$
\begin{aligned}
\frac{\mathrm{d} \sigma\left(\omega_{1}\right)}{\mathrm{d} \omega_{2}}= & k \int_{-\infty}^{\infty} \frac{\omega_{2}}{\omega_{1}} \frac{g_{2 p, 1 s}\left(\Omega_{1 s}-\omega\right)\left(d g_{1 s} / d \omega\right)}{\left(\Omega_{1 s}+\omega-\omega_{1}\right)^{2}+\Gamma_{1 s}^{2} / 4 \hbar^{2}} \\
& \times \delta\left(\omega_{1}-\Omega_{2 p}-\omega-\omega_{2}\right) \mathrm{d} \omega,
\end{aligned}
$$

where

$$
k=2 \pi r_{0}^{2}\left(\Omega_{1 s}-\Omega_{2 p}\right) .
$$

Here $\omega_{1}$ and $\omega_{2}$ are the variable absorption and emission energies, respectively, while $\Omega_{1 s}$ and $\Omega_{2 p}$ are the fixed energies of the $K$-edge and energy loss, respectively. $g_{2 p, 1 s}$ is the oscillator strength of the transition and $\Gamma_{1 s}$ is the lifetime broadening. $d g_{1 s} / d \omega$ is a variable oscillator strength depending on the density of unoccupied states probed in the $1 s$ excitation. The unoccupied states are shifted to $0 \mathrm{eV}$ at the inflection point in the model and the $\delta$ function represents the decay channel. In the case of $1 s 2 p$ RIXS the $\delta$ function can be assumed to be Lorentzian, describing the $K \alpha_{1}$ and $K \alpha_{2}$ decay. For other RIXS experiments, with $K_{\beta}$ or $K_{\text {valence }}$ decay, the Lorentzian decay can be changed to a function of the density of occupied states. This simplified approach has been used to describe hard X-ray RIXS features in $\mathrm{CuO}$ (Hayashi et al., 2002), $\mathrm{LiF}$ (Kikas et al., 2004) and $\mathrm{KMnO}_{4}$ (Tulkii \& Åberg, 1982).

\section{Simple model}

The continuum model equation (2) was applied to an arbitrary system, with an excitation energy at $2000 \mathrm{eV}$ and a decay energy of $1000 \mathrm{eV}$, to illustrate how the convolution in the model effects the decay spectra. Three intuitive cases were explored: (i) a singe excitation, (ii) a square continuum excitation and (iii) a square continuum and an excitation state below the continuum (pre-peak). The RIXS decay spectra, together with the density of unoccpied states (DOS), are shown in Fig. 1. The RIXS decay spectra change by applying different DOS. When the excitation energy is tuned below $2000 \mathrm{eV}$, the spectral intensity drops and, depending on the shape of the DOS, the decay spectra appear distorted. For the model of interest, with a state below the continuum, the decay spectra are strong when tuning the excitation energy to the pre-peak energy. Further, at very low excitation energy the inverted DOS appears, which is applied in the high-energyresolution off-resonant spectroscopy (HEROS) technique (Szlachetko et al., 2012).

\section{Example: vanadium oxide}

Vanadium oxide in the $\mathrm{VO}_{2}$ form has attracted much attention both experimentally (Morin, 1959; Abbate et al., 1991; Shin et al., 1990) and theoretically (Korotin et al., 2002; Liebsch et al., 2002; Haverkort et al., 2005; Yuan et al., 2012; Gatti et al., 2007; Sakuma et al., 2008) due to its metal-insulator transition (MIT) at $67^{\circ} \mathrm{C}$. At room temperature, as in our experiments, the $\mathrm{VO}_{2}$ structure is in the insulating monoclinic phase $\left(\mathrm{M}_{1}\right)$ with an experimental band gap of $0.6 \mathrm{eV}$ (Shin et al., 1990). 


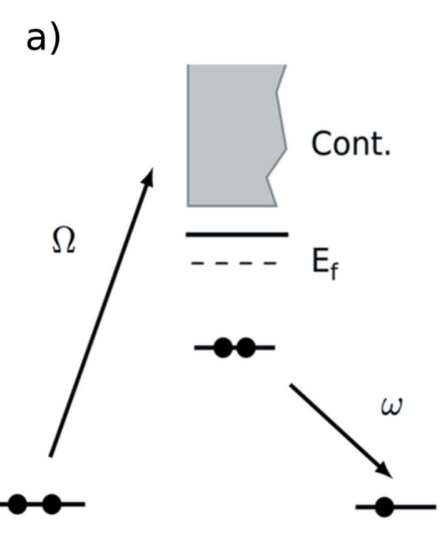

Figure 1

(a) Energy diagram of the RIXS measurement, with $\Omega$ as the excitation photon and $\omega$ as the decay photon. $E_{\mathrm{f}}$ and Cont. denote the Fermi level and the continuum of states, respectively. (b) The continuum model (2) applied to a $2000 \mathrm{eV}$ excitation and $1000 \mathrm{eV}$ decay system. Left: three different density of unoccupied states used as input for the model. Middle: RIXS decay spectra as output from the model at different excitation energies. Right: similar to the middle spectra, but normalized to show all features.

In this experiment the $1 s 2 p$ RIXS data of $\mathrm{VO}_{2}$ were recorded at the BL12XU beamline at SPring-8, Japan. The incident beam was selected by a double-crystal monochromator to a energy bandwidth of less than $1.0 \mathrm{eV}$ at $5480 \mathrm{eV}$. The sample was oriented by $45^{\circ}$ relative to the incident beam. The backscattered fluorescence signal, led through a helium-filled box, was analysed by a silicon (400) crystal. The sample used was a $99.9 \%$ pure Sigma-Aldrich (CAS \#1203621-4) $\mathrm{VO}_{2}$ powder. The experiments were performed at room temperature and with the sample in a nitrogen gas environment to prevent oxidation.

First the $K$-edge XAS of the sample was measured. This was performed by scanning from $40 \mathrm{eV}$ above the $5480 \mathrm{eV}$ edge to $40 \mathrm{eV}$ below. The XAS spectrum is seen in Fig. 2(a). The XAS has been normalized to the incoming photon flux to correct for fluctuations in the synchrotron beam and the background has been subtracted. Further, to apply the XAS as empty DOS in the continuum model, the intensity has been set to 0 at $-5464 \mathrm{eV}$, to remove some of the broadening from the experimental XAS, and last the measured edge value has been extended.

The XAS spectrum shows a pre-peak at $5470 \mathrm{eV}$, which as a first approximation can be assigned to a $1 s \rightarrow 3 d$ transition (quadrupole transition), while the main edge is a $1 s \rightarrow p$-band transition (dipole selection rule). In the $1 s 2 p$ RIXS experiment the emission spectra are measured at specific excitation energies in the pre-peak region (see Fig. $2 d$ ). The RIXS plane shows from left to right the pre-peak and parts of the main edge. In the emission energy the spin-orbit term splits the decay channels into the $K \alpha_{1}$ and $K \alpha_{2}$ decays.

Fig. 2(b) shows the simulated RIXS plane by the continuum model. In this simulation, the RIXS plane is obtained by using the XAS spectra of the $\mathrm{VO}_{2}$ sample as a first approximation for the density of unoccupied states and convoluting it with the Lorentzian $K \alpha_{1}$ and $K \alpha_{2}$ decay channels (originating from spin orbit splitting). The broadening factors were chosen to be $\Gamma_{1 s}=1.5 \mathrm{eV}$ and $\Gamma_{2 p}=0.5 \mathrm{eV}$. In Fig. 2(c) a comparison of a)

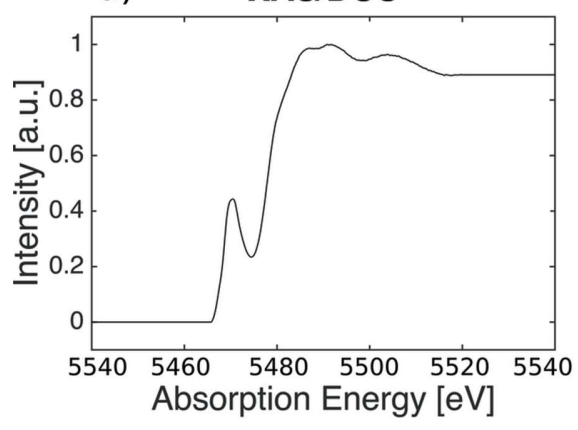

c) Comparison

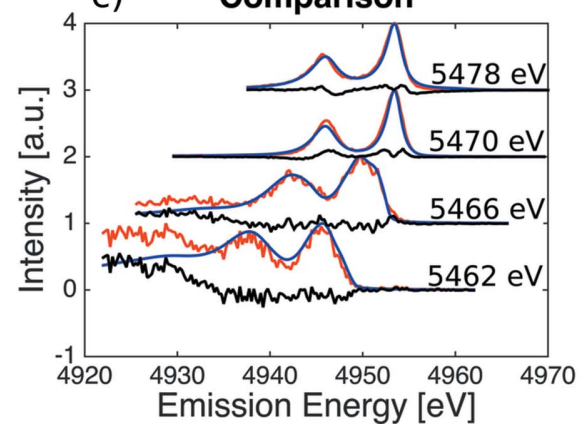

Figure 2

The continuum model applied to the $\mathrm{VO}_{2}$ RIXS experiment. (a) The XAS is used as DOS input for the model. $(b)$ The simulated and $(d)$ experimental RIXS plane. (c) Comparison of selected RIXS slices of the simulation (blue), experiment (red) and difference (black). 


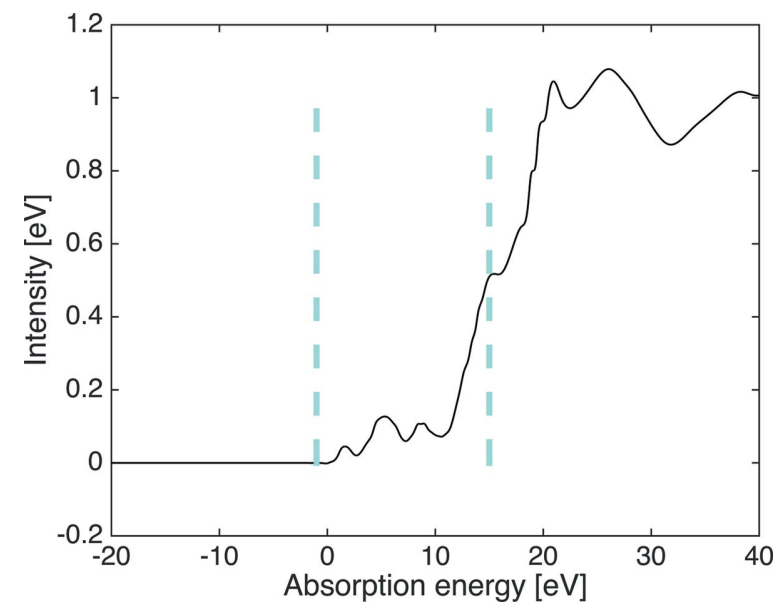

Figure 3

XAS of anatase $\mathrm{TiO}_{2}$. The three pre-peak features are labelled $\mathrm{A}_{1}, \mathrm{~A}_{2}$ and $A_{3}$, where $A_{1}$ is pure quadrupole and $A_{2}$ and $A_{3}$ are dipole. The region of interest for RIXS is within the two dashed lines.

four selected spectra are shown, with the simulation (blue), experiment (red) and difference (black). The blue simulation successfully fits the experiment in this case, both at the edge $(5478 \mathrm{eV})$, in the center of the pre-peak $(5470 \mathrm{eV})$ and at the lower two more distorted RIXS spectra $(5462 \mathrm{eV}$ and $5466 \mathrm{eV})$.

\section{Example: titanium oxide}

$\mathrm{TiO}_{2}$ anatase is interesting for investigation because it has three pre-peak features with both quadrupole and dipole origin (see Fig. 3). The three pre-peak features have been analysed and labelled by Cabaret et al. (1999). The first prepeak, $A_{1}$, is pure quadrupole. The second pre-peak, $A_{2}$, is mainly dipole; however, depending on orientation it has a small quadrupole contribution. The last pre-peak, $\mathrm{A}_{3}$, is a dipole contribution.

In our analysis we want to show how the dipole contribution is simulated by the continuum model and the $A_{1}$, quadrupole, pre-peak is calculated by the exciton model. We therefore import the dipole calculated XAS from Cabaret et al. (1999) and apply it to our simulation, similar to the $\mathrm{VO}_{2}$ example.

Fig. 4(a) shows the dipole RIXS plane and Fig. 4(b) shows the quadrupole RIXS plane. The dipole RIXS plane is simulated by the continuum model via the dipole XAS spectrum. The quadrupole RIXS plane is calculated with the exciton model, using the parameters extracted from the XPS work by Okada \& Kotani (1993) [i.e. DELTA $=4 \mathrm{eV}, U=4 \mathrm{eV}, Q=$ $6 \mathrm{eV}, T(e g)=3 \mathrm{eV}, T(t 2 g)=1.5 \mathrm{eV}$ and $10 \mathrm{Dq}($ ion $)=1.7 \mathrm{eV}]$. For both dipole and quadrupole analysis, we apply the same broadening factors as for the $\mathrm{VO}_{2}$ simulation.

In Fig. 4(c) the dipole simulated and quadrupole calculated RIXS plane are added together. Finally, Fig. 4(d) shows the experimental $1 s 2 p \mathrm{TiO}_{2}$ RIXS plane [data courtesy of Glatzel et al. (Kas et al., 2011; Glatzel et al., 2013)]. In the bottom row for each RIXS plane three selected emission spectra are shown: $4965 \mathrm{eV}$ (red), $4967 \mathrm{eV}$ (blue) and $4970 \mathrm{eV}$ (black).

The combined RIXS plane and the three selected RIXS spectra in the bottom row are identical to the experimental RIXS result. This is also illustrated in Fig. 5, where a direct comparison of the experimental and simulated RIXS spectra has been carried out. We do not show a difference curve, as in X-ray diffraction data or alike, because this requires a fitting of both broadening factors and possible energy shifts. Here it is seen that peak position and shape compare nicely with experimental data. This is a strong indication that the methodology of calculating the dipole and quadrupole RIXS planes separately is a good approach. Furthermore, in this method, each peak can be assigned to quadrupole or dipole origin. In the RIXS spectra at $4965 \mathrm{eV}$ (red), the central peak is a dipole
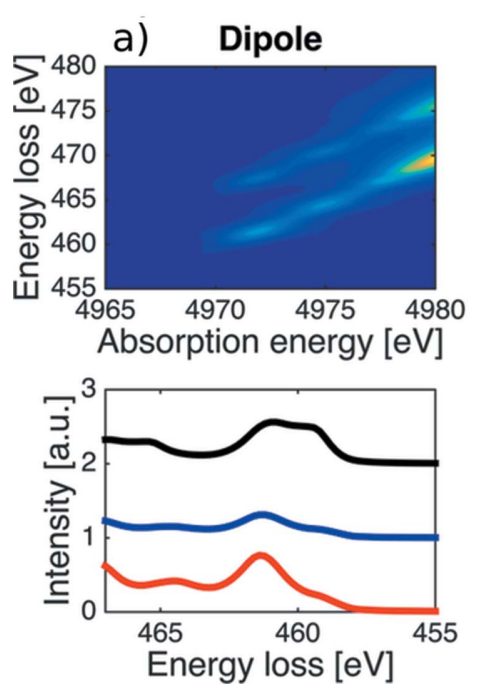
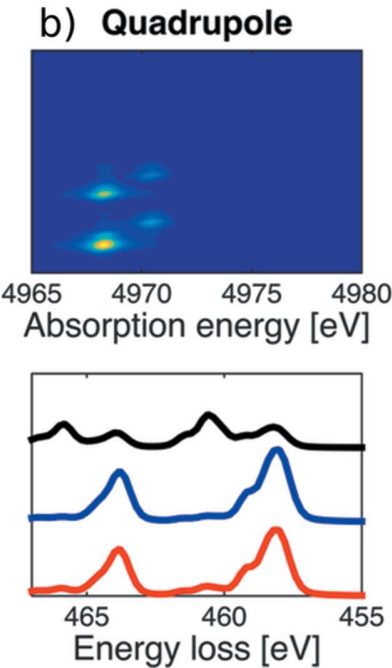

c) Dipole + Quadrupole
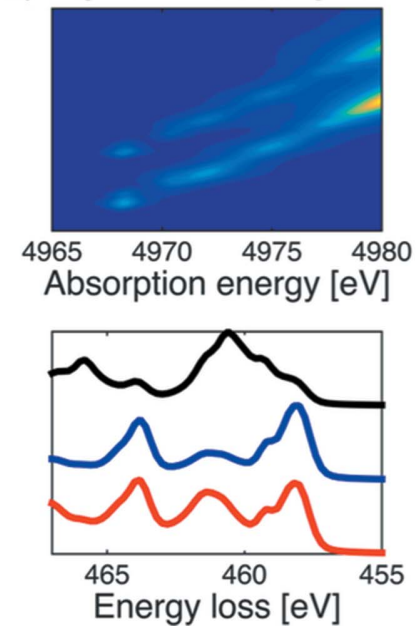

\section{d) Experiment}
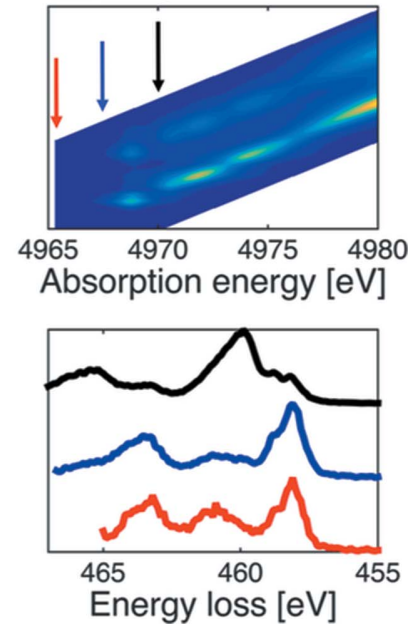

Figure 4

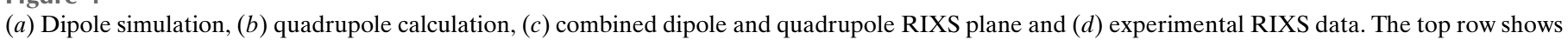

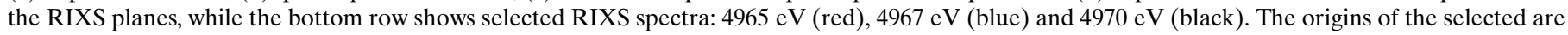

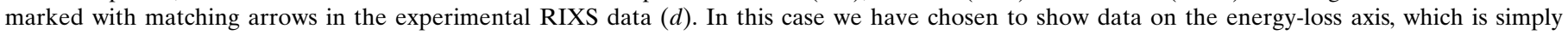
calculated by subtracting the emission energy from the absorption energy. 


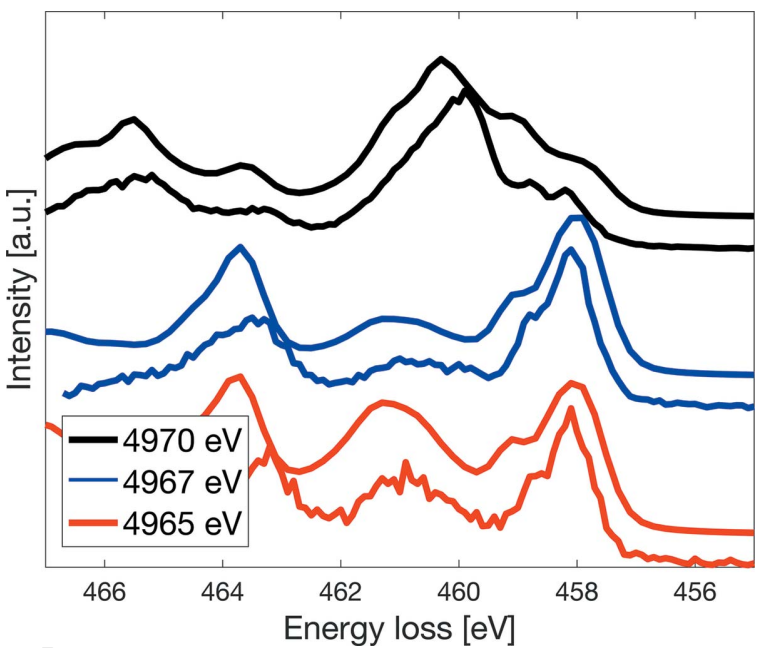

Figure 5

Direct comparison between RIXS spectra $4965 \mathrm{eV}$ (red), $4967 \mathrm{eV}$ (blue) and $4970 \mathrm{eV}$ (black) from Figs. 4(c) and 4(d) bottom. The simulation is placed just above the experimental spectra.

peak from the continuum model, while the left and right peaks with shoulder features are quadrupoles. Also we identify the quadrupole double peak on the diagonal of the pure quadrupole RIXS plane as found by Cabaret et al. (1999).

\section{Discussion}

In the previous two sections we have displayed how the $\mathrm{VO}_{2}$ experimental RIXS plane can be simulated entirely by the continuum model. Furthermore, the continuum model describes the two dipole peaks of the $\mathrm{TiO}_{2}$ anatase RIXS experiment. However, the quadrupole pre-peak of $\mathrm{TiO}_{2}$ requires the exciton model to be explained.

In the continuum model a single electron excitation approximation is applied, which means that the model describes excitations to band-like unoccupied states. Compared with the difficulties of describing the correct band structure of the MIT of $\mathrm{VO}_{2}$ it is quite remarkable that at the current resolution of less than $1.0 \mathrm{eV}$ the continuum model actually achieves this good description with the current experimental resolution.

In particular, in the case of exciting to band-like states, it must be realised that it is not necessary to measure the RIXS plane explicitly as it can be generated by the convolution of the XAS and the XES (Rubensson, 2000).

If an already known analysis of the XAS pre-peak region exists, as in the case of $\mathrm{TiO}_{2}$ by Cabaret et al. (1999) or the $\mathrm{VOPO}_{4} \cdot 2 \mathrm{H}_{2} \mathrm{O}$ analysis by Poumellec et al. (1998), then, beforehand, the area of interest can be defined and measured by RIXS. However, if the pre-peak region is unknown, we suggest, for pure systems, the following steps:

(i) Measure the XAS and XES spectra, and create the twodimensional RIXS plane by the convolution of the XAS and XES spectra.

(ii) Measure some RIXS slices. Note that if the RIXS spectra are equal to the convolution, one cannot achieve further insight from RIXS experiments. (iii) If the RIXS slices deviate from the convolution, this quadrupole RIXS pre-edge region should be measured in a RIXS experiment and at a high resolution.

In the case of mixed systems it could still be beneficial to measure selected parts of the two-dimensional RIXS planes, to increase the accuracy in distinguishing different phases/ valences/etc. Additionally, we believe that, in time, with increased resolution, exciton features will appear for all $d$ and $f$ systems as seen for the CoO RIXS investigation by Kurian et al. (2013).

In this paper we only investigated $1 s 2 p$ RIXS of high valence compounds and it is interesting to think about how the methodology is extended to experiments other than $1 s 2 p$ RIXS. For $L_{2,3}$ RIXS the decay may not be a simple Lorentzian decay as currently applied in the continuum model. Instead, the Lorentzian can be replaced by the density of occupied states (Smolentsev et al., 2011). Another important point is that, with poor resolution, every RIXS spectrum can be described with the convolution model. In other words, one needs an excitonic effect that is larger than the experimental resolution in order to become visible. This may limit the usage of the convolution model with the present resolution of soft $\mathrm{X}$-ray beamlines ranging from 20 to $200 \mathrm{meV}$ for most systems.

\section{Conclusion}

In this work we have presented and applied two models for analysing RIXS data: (i) the exciton model and (ii) the continuum model. We have shown how RIXS features of dipole origin can be simulated by a convolution of the XAS and XES spectra (continuum model). Additionally, we have shown that the continuum model can describe all features of $1 s 2 p \mathrm{VO}_{2}$ experimental RIXS plane. Furthermore, the analysis of the $\mathrm{TiO}_{2}$ RIXS plane, with a combination of the continuum and exciton models, shows that only quadrupole RIXS peaks are relevant for RIXS measurements, while for dipole peaks a XAS experiment will be sufficient.

\section{Acknowledgements}

The Innovation Fund Denmark (Ties Haarman Industrial $\mathrm{PhD}$ ) is gratefully acknowledged for financial support. Further, we thank Pieter Glatzel for $\mathrm{TiO}_{2}$ data and discussion.

\section{References}

Abbate, M., de Groot, F. M. F., Fuggle, J. C., Ma, Y. J., Chen, C. T., Sette, F., Fujimori, A., Ueda, Y. \& Kosuge, K. (1991). Phys. Rev. B, 43, 7263-7266.

Ament, L. J. P., van Veenendaal, M., Devereaux, T. P., Hill, J. P. \& van den Brink, J. (2011). Rev. Mod. Phys. 83, 705-767.

Cabaret, D., Joly, Y., Renevier, H. \& Natoli, C. R. (1999). J. Synchrotron Rad. 6, 258-260.

De Groot, F. \& Kotani, A. (2008). Core Level Spectroscopy of Solids, Vol. 1, 1st ed. Boca Raton: CRC Press.

Garino, C., Gallo, E., Smolentsec, N., Glatzel, P., Gobetto, R., Lamberti, C., Sadler, P. J. \& Salassa, L. (2012). Phys. Chem. 14, $15278-15281$. 
Gatti, M., Bruneval, F., Olevano, V. \& Reining, L. (2007). Phys. Rev. Lett. 99, 266402.

Glatzel, P., Singh, J., Kvashnina, K. O. \& van Bokhoven, J. A. (2010). J. Am. Chem. Soc. 132, 2555-2557.

Glatzel, P., Weng, T., Kvashnina, K., Swarbrick, J., Sikora, M., Gallo, E., Smolentsev, N. \& Mori, R. A. (2013). J. Electron Spectrosc. Relat. Phenom. 188, 17-25.

Groot, F. M. F. de, Glatzel, P., Bergmann, U., van Aken, P. A., Barrea, R. A., Klemme, S., Hävecker, M., Knop-Gericke, A., Heijboer, W. M. \& Weckhuysen, B. M. (2005). J. Phys. Chem. B, 109, 2075120762.

Haverkort, M. W., Hu, Z., Tanaka, A., Reichelt, W., Streltsov, S. V., Korotin, M. A., Anisimov, V. I., Hsieh, H. H., Lin, H.-J., Chen, C. T., Khomskii, D. I. \& Tjeng, L. H. (2005). Phys. Rev. Lett. 95, 196404.

Hayashi, H., Udagawa, Y., Caliebe, W. A. \& Kao, C.-C. (2002). Phys. Rev. B, 66, 033105.

Juhin, A., de Groot, F., Vankó, G., Calandra, M. \& Brouder, C. (2010). Phys. Rev. B, 81, 115115.

Kas, J. J., Rehr, J. J., Soininen, J. A. \& Glatzel, P. (2011). Phys. Rev. B, $\mathbf{8 3}, 235114$.

Kikas, A., Käämbre, T., Saar, A., Kooser, K., Nõmmiste, E., Martinson, I., Kimberg, V., Polyutov, S. \& Gel'mukhanov, F. (2004). Phys. Rev. B, 70, 085102.

Korotin, M. A., Skorikov, N. A. \& Anisimov, V. I. (2002). Phys. Metals Metallogr. 94, 17-23.
Kurian, R., van Schooneveld, M. M., Zoltan, N., Vanko, G. \& de Groot, F. (2013). Phys. Chem. 117, 2976-2981.

Liebsch, A., Ishida, H. \& Bihlmayer, G. (2002). Phys. Rev. B, 71, 089109.

Morin, F. J. (1959). Phys. Rev. Lett. 3, 34-36.

Okada, K. \& Kotani, A. (1993). J. Electron Spectrosc. Relat. Phenom. 62, 131-140.

Poumellec, B., Kraizman, V., Aifa, Y., Cortès, R., Novakovich, A. \& Vedrinskii, R. (1998). Phys. Rev. B, 58, 6133-6146.

Rubensson, J.-E. (2000). J. Electron Spectrosc. Relat. Phenom. 110111, 135-151.

Sakuma, R., Miyake, T. \& Aryasetiawan, F. (2008). Phys. Rev. B, 78, 075106.

Shin, S., Suga, S., Taniguchi, M., Fujisawa, M., Kanzaki, H., Fujimori, A., Daimon, H., Ueda, Y., Kosuge, K. \& Kachi, S. (1990). Phys. Rev. $B$, 41, 4993-5009.

Smolentsev, N., Sikora, M., Soldatov, A. V., Kvashnina, K. O. \& Glatzel, P. (2011). Phys. Rev. B, 84, 235113.

Stavitski, E. \& de Groot, F. (2010). Micron, 41, 687-694.

Szlachetko, J., Nachtegaal, M., Sa, J., Dousse, J.-C., Hoszowska, J., Kleymenov, E., Janousch, M., Safonova, O. V., König, C. \& van Bokhoven, J. A. (2012). Chem. Commun. 48, 10898-10900.

Tulkki, J. \& Åberg, T. (1982). J. Phys. 15, L435-L440.

Yuan, X., Zhang, Y., Abtew, T. A., Zhang, P. \& Zhang, W. (2012). Phys. Rev. B, 86, 235103. 